\title{
Mixed method design in and for Asian migration research
}

\author{
Hyekyung Choo
}

Published online: 5 June 2014

(C) Springer Science+Business Media Dordrecht 2014

The first two papers in this issue of the Journal of Population Research focus on using mixed-method designs for investigating Asian migration research. For the past few decades, the adoption and application of traditional migration research methods derived from the experience of Western countries have manifested numerous methodological challenges in research on migration in Asia. Particularly in the quantitative approach to Asian migration research, methodological limitations include the inappropriate conceptualization of migration in Asian contexts, the exclusive focus on the overall flow of international migrant population at the national level, and the heavy use of secondary, demographic data, rather than primary data. While quantitative research on Asian migration is very much needed to produce comparable data across countries and cultures with the enhanced generalizability of findings for policy-relevant implications, its utility is often compromised when it comes to reaching out to newly emerging groups of migrants in Asia, and employing culturally relevant measurements and data gathering in the Asian context. It is probably there that the quantitative approach and qualitative approach should meet strategically. However, mixed-method design sometimes may turn out any haphazard combination of the two methods. Although sounding good theoretically, the use of mixed methods may create tensions in implementation, especially if one method rather than the other is added later to a study in an ad hoc fashion, not streamlined in its designing stage. Therefore, the deliberate planning and executing of mixed-method research is crucial in building a comprehensive yet coherent body of knowledge, and this applies equally to Asian migration research.

Asian migration research explores a broad range of new phenomena, many of which have rarely been observed in Asian migration within non-Asian societies.

H. Choo (ه)

National University of Singapore, Singapore, Singapore

e-mail: swkch@nus.edu.sg 
More and more diverse groups of Asians migrate from one Asian country to another for various incentives and prospects. Their accommodation, assimilation, or integration not only significantly differs from those experienced in the Western context, but also becomes increasingly fluid owing to the geographical and cultural proximity between their home and host countries. Capturing such trends at macro levels would certainly require large-scale surveys or demographic data. Without the contextual understanding of the key constructs of quantitative data to be examined, however, it would be difficult in Asian migration research to formulate meaningful and relevant study hypotheses, identify suitable questions and instruments for data collection, make sense out of unexpected findings from quantitative data, and set future research directions. In this regard, the contribution of contextual knowledge gained through qualitative investigations is more than complementary to quantitative research. Mixed methods are not new, but their potential usefulness in Asian migration research is anticipated to receive increasingly wide recognition.

The following two articles present some methodological challenges and merits of mixed-method approaches in recent migration research on two youth populations in Asia. Y. Ge and K. C. Ho's article, 'Researching international student migration in Asia: Research design and project management issues' is a systematic, behind-the-scenes elaboration of the process of conducting their mixed-method study with Asian international students in Asia. Despite the complexity of this multifaceted research, which involves not only both quantitative and qualitative approaches, but also multisited, multi-institutional, and multilingual data collection, the authors coherently explain how the interface between the survey method and the biographical interview approach facilitates effective collection of data and management of overall research projects. New considerations, such as cultural contexts related to provision of tokens of appreciation and engagement of interviewers, and other lessons for future research on Asian international students can be inferred from their detailed experiences. This study is indeed a good illustration of how the authors' concluding remarks come about: 'A good research design will thus be the one that is flexible enough to be altered should the need arise, and yet still be able to fulfil the original objectives of the research'.

Y. Chung and J. Mohanty's paper, 'Between two worlds in Asia: Korean youth in Singapore' is a successful example of a strategic use of the qualitative method before the quantitative approach. Using the exploratory sequential mixedmethod design proposed by Creswell and Plano Clark (2007), the study demonstrates how quantitative research can be guided by, and builds on preceding qualitative findings within the same study. Initial findings from qualitative data focus on how the cultural competency of Korean youth in Singapore-Asian migrants in an Asian host society-develops and is negotiated, and how their assimilation differs from that of their counterparts in non-Asian host societies. At the same time, the researchers effectively incorporate these qualitative results into the next stage of research using the quantitative approach in three significant ways: based on the qualitative findings, they formulate study hypotheses for the subsequent quantitative investigation, suggest specific changes 
to be made in the common constructs of established instruments of assimilation stress, and highlight the importance of 'the context of reception' as a key consideration for Asian migration research.

\section{Reference}

Creswell, J. W., \& Plano Clark, V. L. (2007). Design and conducting mixed methods research. Thousand Oaks: Sage. 